\title{
ARITHMETIC ON OPEN RIEMANN SURFACES
}

\author{
KARL EGIL AUBERT
}

\section{Introduction}

An abstract theory of divisibility in ordered groups has evolved out of developments which initially started in algebraic number theory and later gave rise to a more general arithmetical theory of integral domains.

The third, and probably final, phase in this development of purifying the notion of divisibility is above all represented by the work of Paul Lorenzen, especially in his 1939 thesis [14]. After some forerunners in the works of Arnold [2] and Clifford [7], Lorenzen created a full-fledged multiplicative theory of divisibility, freed from any intervention of an extraneous additive operation. Thus, divisibility theory finally found its natural home in the setting of ordered groups.

In [4] we tried to bring together various aspects of the crucial role which is played by the divisors of finite character (also called $t$-ideals) in the arithmetical study of ordered groups. In view of the interests of the man we are honouring with the present volume, I would like to give a preliminary report on the possible relevance of ideal systems and $t$-ideals in the context of open Riemann surfaces.

\section{Ideal systems and $t$-ideals}

Given an integral domain $R$ with $K$ as its field of quotients, we form the factor group $G=K^{*} / U$, where $K^{*}=K-\{0\}$ is the multiplicative group of $K$ and $U$ is the group of units in $R$. This factor group is nothing but the group of non-zero fractional principal ideals in $K$. Considering $G$ as an ordered (directed) group with respect to divisibility $a \leqq b \Leftrightarrow a \mid b \Leftrightarrow(b) \subset(a), G$ is termed the group of divisibility of $R$. The fact is that all the essential divisibility properties of $R$ are reflected in the structure of $G$, which prompts our shift of interest from $R$ to an arbitrary (abelian and directed) ordered group $G$. In this latter generality it is the monoid $D=G^{+}=\{g \mid g \geqq e\}$ of integral (positive) elements which corresponds to $R$. That $G$ is the group of quotients of $D$ is equivalent to saying that $G$ is directed (i.e., there exists a $c \in G$ such that $a \leqq c$ and $b \leqq c$ corresponding to any two elements $a, b \in G)$.

The classical problem of adjoining ideals or divisors in order to achieve a nicer theory of divisibility corresponds in this purely multiplicative setting to the question 
of when and how a directed group $G$ can be isomorphically injected into a GCDgroup or into a factorial group. By a GCD-group we mean a group which is lattice ordered, i.e., which reflects the property of possessing greatest common divisors. A factorial group is a GCD-group of the form $Z^{(I)}-$ an ordered direct sum of copies of $\boldsymbol{Z}$ (a free abelian group with pointwise order).

The solution of the above completion problems depends heavily on the theory of ideal systems. An ideal system (in the sense of Lorenzen [14]) is defined in the directed abelian group $G$ if there exists a set $A_{x} \subset G$ (called the $x$-ideal generated by $A$ ) such that
(1) $A \subset A_{x}$,
(2) $B \subset A_{x} \Rightarrow B_{x} \subset A_{x}$,
(3) $\{a\}_{x}=(a)=D a$,
(4) $a A_{x}=(a A)$

corresponding to any subset $A$ of $G$ which is bounded from below.

The most interesting ideal systems are those which are of finite character, i.e., satisfy

$$
A_{x}=\bigcup_{\substack{N \subset A \\ N \text { finite }}} N_{x} .
$$

An ideal system $A \rightarrow A_{x}$ in $G$ is said to be finer than another ideal system $A \rightarrow A_{y}$ in $G$ if $A_{x} \subset A_{y}$ for all $A$, or, equivalently, if every $y$-ideal in $G$ is an $x$-ideal in $G$. It is easily seen that there exists one ideal system, the so-called s-system defined by $A_{s}=D A$, which is finer than all the others, as well as the $v$-system defined by

$$
A_{v}=\bigcup_{A \subset(a)}(a),
$$

which is coarser than all the other systems in $G$. The $v$-ideal $A_{v}$ may equivalently be given by $A_{v}=D:(D: A)$ and is sometimes called the divisorial ideal generated by $A$. (In [6] $A_{v}$ is simply called a divisor.) The $v$-system is generally not of finite character. However, there exists a unique coarsest ideal system of finite character in $G$, called the $t$-system. The $t$-ideal (divisor of finite character) generated by $A$ is the set-theoretic union of all the $v$-ideals generated by finite subsets of $A$ :

$$
A_{t}=\underset{\substack{N \subset A \\ N \text { finite }}}{\bigcup} N_{v} .
$$

If $G$ is a GCD-group with the GCD-operation (intersection) denoted by $\wedge$, the definition of a $t$-ideal $A_{t}$ assumes a more appealing form as the conjunction of the two properties

$$
D A_{t} \subset A_{t} \text { and } a, b \in A_{t} \Rightarrow a \wedge b \in A_{t} .
$$

In the case of the group of divisibility $G=K^{*} / U$ of an integral domain, $G$ also carries a fourth distinguished ideal system consisting of all the canonical images in $G$ (relative to the map $K^{*} \rightarrow K^{*} / U$ ) of all the non-zero fractional ideals in $K$. This is the system of $d$-ideals in $G$ ( $d$ for Dedekind). 
Among all ideal systems the $t$-ideals occupy a distinguished arithmetical role due to the following facts:

1. $G$ is a GCD-group if and only if $G$ is $t$-Bezout, i.e., if and only if every finitely generated $t$-ideal in $G$ is principal.

2. $G$ is a factorial group if and only if every $t$-ideal in $G$ is principal.

3. $G$ is a préfactorial group, i.e., it can be embedded as an ordered subgroup of a factorial group if and only if $G$ is a $t$-Dedekind group meaning that the fractional $t$-ideals of $G$ form a group under $t$-multiplication (and this latter group then forms the desired (minimal) factorial extension).

The properties 2 . and 3 . show that the $t$-ideals restore unique factorization in an optimal way: If unique factorization can at all be restored by some extension process, this can also be done by means of $t$-ideals and then in a most economical (minimal) way.

\section{Commutative algebra of ideal systems}

The point that we want to make in the present paper is that there is something to be gained by using the general commutative algebra of ideal systems in the group of divisibility of the ring of entire functions on an open Riemann surface $X$, instead of staying within the more restricted realm of the classical commutative algebra related solely to the $d$-system. Each ideal system (of finite character) gives rise to a bundle of concepts of commutative algebra like noetherianness, integral closure, valuation, localization, spectrum etc. For the sake of illustration let us pick three of these notions: integral closure, valuation and localization, and spell them out in the case of the $t$-system.

The group $G$ is said to be (integrally) $t$-closed if $A_{t}: A_{t} \subset D=G^{+}$for any finite set $A \subset G$. This definition derives its naturalness from the fact that an integral domain $R$ is integrally closed in its field of quotients if and only if $A_{d}: A_{d} \subset R$ for any finite subset $A$ of the field of quotients of $R$ (or, rather, $A_{d}: A_{d} \subset G^{+}$when considering the $d$-system in the group of divisibility of $R$ ). It is easily seen that a GCD-group is always integrally $t$-closed.

A $t$-valuation of a general directed group $G$ is a homomorphism of ordered groups of $G$ onto a totally (linearly) ordered group $\Gamma$ such that the inverse image of a $t$-ideal in $\Gamma$ is a $t$-ideal in $G$. Again, this concept derives its naturalness and relevance from the fact that the classical notion of a Krull valuation is obtained by replacing $G$ by the group of divisibility of an integral domain and requiring that inverse images of $t$-ideals are $d$-ideals.

The notion of localization with respect to a prime ideal in a given ideal system (of finite character) in a directed group $G$ is also quite natural and in the case of the $t$-system in a GCD-group assumes a particularly appealing form. (For the basics of the general theory see [3].) Let $P_{t}$ be a prime $t$-ideal in the monoid $D$ of integral 
elements of a GCD-group $G$, put $S=D-P_{t}$ and introduce the 'localized monoid' $S^{-1} D$ consisting of all the elements in $G$ which are of the form $d s^{-1}$ with $d \in D$ and $s \in S$. If we take $S^{-1} D \supset D$ as a new monoid of integral elements in $G$, this will induce a new preorder in $G\left(a \leqq b \Leftrightarrow b a^{-1} \in S^{-1} D\right)$ and finally give rise to a corresponding ordered group by factoring out the units of $S^{-1} D$. This group of units is $U=S^{-1} D \bigcap S D^{-1}$. Since $U$ is closed under the GCD-operation in the original order of $G$ (and is hence a so-called absolutely or lattice-closed convex subgroup of $G$ ), the factor group $G / U$ is a GCD-group relative to the order which is obtained by taking the monoid $S^{-1} D / U$ as a monoid of integral elements in $G$. This GCD-group is termed the localization of $G$ at $P_{t}$. Lorenzen proved in [14] (Satz 10) the simple but useful theorem that the localizations of a GCD-group at prime $t$-ideals are not only GCD-groups but are in fact totally ordered.

As in the classical commutative algebra (which is based on the $d$-system), there are various interrelations between the concepts of $t$-closure, $t$-valuation and $t$-localization. In particular, $D=G^{+}$is $t$-closed in $G$ if and only if $D$ appears as an intersection of $t$-valuation monoids (a $t$-valuation monoid being the set of elements in $G$ which by a $t$-valuation $G \rightarrow \Gamma$ is mapped onto the positive (integral) part of $\Gamma$ ). Furthermore, one can define $t$-localizations also in the case of directed groups $G$ which are not GCD-groups and for instance establish that the finitely generated (fractional) $t$-ideals of $G$ form a group under $t$-multiplication (i.e., $G$ is a $t$-Prüfer group) if and only if all the localizations at prime $t$-ideals of $G^{+}$are totally ordered.

\section{The group of divisibility of an open Riemann surface}

Much attention has been paid to the study of the algebraic structure of the ring $A(X)$ of all entire functions on an open Riemann surface $X$ as well as to the field $M(X)$ of all meromorphic functions on $X$. Much of this research has been connected with the problem of recovering the analytic structure of $X$ from the algebraic structure of either $A(X)$ of $M(X)$. Among the names associated with these studies are Heins, Royden, Rudin, Bers, Nakai, Kakutani, Kra, Banachewski [5], Alling [1] and Hej Iss'sa [12].

We shall refer to the group of divisibility $G(X)$ of the ring $A(X)$ as the group of divisibility of the Riemann surface $X . G(X)$ is an ordered group consisting of all the non zero principal fractional ideals in $M(X)$ putting $(f) \leqq(g)$ whenever $f g^{-1}$ is an entire function on $X$. In order to describe the structure of $G(X)$ it is convenient to introduce the notion of a divisor on $X$ as a mapping of $X$ into the integers $Z$ whose restriction to any compact set has a finite support. Under pointwise addition and ordering the family of all divisors on $X$ form an ordered group which we denote by $\boldsymbol{Z}^{[X]}$. (We have $\boldsymbol{Z}^{(X)} \subset \boldsymbol{Z}^{[X]} \subset \boldsymbol{Z}^{X}$, where $\boldsymbol{Z}^{(X)}$ denotes the factorial group of all divisors on $X$ with finite support.)

The main result on $G(X)$ is of course 
Theorem 1 (Weierstrass-Florack). The group of divisibility of an open Riemann surface $X$ is isomorphic to the ordered group $Z^{[X]}$ of all divisors on $X$.

Whereas $A(X)$ and $M(X)$ both determine $X$ up to a conformal isomorphism or anti-isomorphism (see [12]), it was established by Alling [1] that $G(X)$ and $G(Y)$ are always isomorphic as GCD-groups for any pair of (connected) open Riemann surfaces $X$ and $Y$, showing that $G(X)$ carries no analytic or topological content. Nevertheless, $G(X)$ and the commutative algebra related to its various ideal systems proves to be helpful also for the study of $A(X)$ as a ring and of $M(X)$ as a field. Some of these applications are quite simple but have an interest in a historical perspective. Before taking up this topic we ought to mention one other basic result on the structure of $A(X)$, namely, the Wedderburn-Helmer theorem that $A(X)$ is a Bezout ring, i.e., that any finitely generated ideal in $A(X)$ is principal. When transferred to $G(X)$ this theorem may be formulated as follows in the language of ideal systems:

Theorem 2 (Wedderburn-Helmer). The d-system in $G(X)$ coincides with the t-system.

Proof: For any group of divisibility of an integral domain it is clear that all $t$-ideals are $d$-ideals. Conversely, given a $d$-ideal in $G(X)$ and two elements $a, b \in A_{d}$, we can by the original result of Wedderburn-Helmer (for arbitrary $X$ ) conclude that the greatest common divisor $c=a \wedge b$ is in $A_{d}$ and hence that $A_{d}$ is a $t$-ideal.

The fact that $G(X)$ is a GCD-group assures us that any finitely generated $t$-ideal in $G(X)$ is principal and hence that the above theorem implies the classical formulation of 'Helmer's lemma', i.e., that $A(X)$ is a Bezout ring.

\section{Some historical observations}

The notion of a $t$-ideal is already implicit in the work of Banachewski [5] and Alling [1] in the form of 'dual ideals'. However, in their treatment ideals and dual ideals ( $d$-ideals and $t$-ideals) are given the place of subobjects of different algebraic objects. In our treatment both the $d$-ideals and the $t$-ideals appear as subobjects of $G(X)$, a fact which facilitates the comparison between them. Indeed, the more complicated bijection which Banachewski and Alling set up between ideals and dual ideals is in our formulation a triviality by our alternative version of the Wedderburn-Helmer theorem (Theorem 2), which simply reduces this bijection to the identity map in $G(X)$.

The theorem that Alling refers to as Banachewski's Theorem (p. 248 in [1]) to the effect that the bijection between ideals and dual ideals preserves the properties of being maximal, prime or primary, appears in our presentation as something which is already contained in Theorem 2 . In fact, it is this theorem that is at the root of the following statement of Banachewski (as translated by Alling): "The 
multiplicative ideal theory of $A(X)$ is hence identical with the dual ideal theory of Div ${ }^{+} X "$.

More important than the formal simplification achieved by identifying ideals and dual ideals ( $d$-ideals and $t$-ideals) as one and the same ideal system in $G(X)$ is to take advantage of the nice calculus of $t$-ideals. The main bulk of the following remarks pertains to this subject.

In the introduction to his paper [1] Alling mentions three classical results on the algebraic structure of $A(\boldsymbol{C}): A(\boldsymbol{C})$ is a Bezout ring (Wedderburn-Helmer $1915,1940)$, every prime ideal of $A(\boldsymbol{C})$ is contained in a unique maximal ideal (Henriksen, 1953) and every localization at a maximal ideal of $A(\boldsymbol{C})$ is a valuation ring (Alling 1963).

It is interesting that at least two of these results were essentially available in the literature since the publication of Lorenzen's thesis [14] in 1939, prior to the publication of Helmer's 1940 paper, which by everybody has been considered as the pioneering work on the arithmetic of $A(X)$. I am indebted to the referee for calling my attention to the paper [15] by J. H. M. Wedderburn, published in 1915, and essentially containing a proof of the fact that $A(C)$ is a Bezout ring ('Helmer's lemma'). Apparently, the referee has learned this in a memorandum circulated by Professor Melvin Henriksen in 1979.

We have already mentioned 'Satz 10' of Lorenzen's 1939 paper stating that any localization of a GCD-group at a prime $t$-ideal is totally ordered. If we combine this result with Wedderburn's result (Theorem 2) of 1915, we immediately obtain the result contained in Alling's 1963 paper referred to above (even in the somewhat strengthened form which he gives in Lemma 3.6 in his more recent paper [1]). In fact, the Wedderburn-Helmer theorem tells us that the localization at a prime $d$-ideal is the same as the localization at a prime $t$-ideal, the latter being totally ordered in view of Lorenzen's theorem.

In 1953 Henriksen [11] showed that the prime ideals contained in a maximal ideal of $A(X)$ are totally ordered by set inclusion. After it has been established that all the localizations at prime ideals of $A=A(X)$ are valuation rings, this follows most easily by the fact that there is a one-to-one inclusion-preserving correspondence between the prime ideals of $A_{M}$ and those prime ideals of $A$ which are contained in $M$. In accordance with the philosophy of the present paper I should also like to present a simple proof of Henriksen's result within the context of ordered groups.

So, using the group of divisibility $G(X)$ instead of dealing directly with $A(X)$, we assume that $M_{t}$ is a maximal (integral) $d$-ideal in $G(X)$ and that $P_{t}^{(1)}$ and $P_{t}^{(2)}$ are two prime $d$-ideals contained in $M_{t}$. By using the subscript $t$ we have already indicated that these $d$-ideals are actually $t$-ideals (Theorem 2). Using the same notation as in Paragraph 3 we put $D=G(X)^{+}, S=D-M_{t}, S_{1}=D-P_{t}^{(1)}, S_{2}=D-P_{t}^{(2)}$, $U=S^{-1} D \cap S D^{-1}, U_{1}=S_{1}^{-1} D \cap S_{1} D^{-1}$ and $U_{2}=S_{2}^{-1} D \cap S_{2} D^{-1}$. Clearly $U \subset U_{1} \cap U_{2}$ and the factor groups $U_{1} / U$ and $U_{2} / U$ are absolutely convex subgroups in the factor group $G(X) / U$. Since this latter group is isomorphic to the localization of $G(X)$ at 
$M_{t}$ and hence is totally ordered by Lorenzen's theorem, it follows that $U_{1} / U \subset U_{2} / U$ or $U_{2} / U \subset U_{1} / U$ (since the convex subgroups of a totally ordered group are totally ordered by set inclusion). Furthermore, this entails that $U_{1} \subset U_{2}$ or $U_{2} \subset U_{1}$, which in turn implies $P_{t}^{(1)} \subset P_{t}^{(2)}$ or $P_{t}^{(2)} \subset P_{t}^{(1)}$ as desired.

Those papers on $A(X)$ which were written before 1963 could not easily make use of the nice characterizations of Prüfer rings which appeared in Jensen's paper [13]. Since any Bezout ring is a Prüfer ring, these characterizations apply immediately to $A(X)$. It follows not only that all the localizations at prime ideals of $A(X)$ are valuation rings but also that $A(X)$ has a distributive ideal lattice and that the Chinese remainder theorem holds in $A(X)$ - as well as many other properties (see for instance [6], pp. 558-559, for a much longer list of properties shared by any Prüfer ring).

Taking [4] into account we could easily add a few more algebraic properties of $A(X)$ or $G(X)$ to the already existing list. By Corollary 1 of Theorem 7 of that paper it follows that $G(X)$ may be represented as the GCD-group of all sections in a sheaf of totally ordered groups over the quasi-compact space of prime $t$-ideals under the spectral (Zariski) topology. From Theorem 8 of [4] it follows furthermore that the $t$-prime spectrum of $G(X)$ is homeomorphic to the $t$-valuation spectrum of $G(X)$, a property which is closely related to the fact that $A(X)$ is a $t$-Prüfer ring.

\section{Krull valuations of $M(X)$ are $t$-valuations of $G(X)$}

The above considerations have centered around the applicability of $t$-ideals to the algebraic study of $A(X)$. Let us also mention one such result which concerns the field $M(X)$ rather than the ring $A(X)$.

In Paragraph 3 we defined the notion of a $t$-valuation of a directed group $G$ as a homomorphism of ordered groups of $G$ onto a totally ordered group $\Gamma$ such that the inverse image of a $t$-ideal in $\Gamma$ is a $t$-ideal in $G$ (for information on the general concept of an $x$-valuation see [4]). It turns out that the notion of a $t$-valuation may be used in order to give a characterization of those Krull valuations of $M(X)$ which are positive on $A(X)-\{0\}$ solely in terms of multiplicative and order-theoretic properties (i.e., not involving the additive structure of $M(X)$ or the $d$-system in $G(X))$ :

Theorem 3. The Krull valuations of the field $M(X)$ which are positive on $A(X)-\{0\}$ correspond one-to-one to the family of all the homomorphisms of GCDgroups of $G(X)$ onto totally ordered groups $\Gamma$.

Proof: First of all, the property $v(a \pm b) \geqq \operatorname{Min}(v(a), v(b))$ for a Krull valuation $v$ with value group $\Gamma$ (and being positive on $A(X)-\{0\}$ ) is easily seen to amount to the fact that inverse images (relative to $v$ ) of $t$-ideals in $\Gamma$ are $d$-ideals of $M(X)-$ or, if you will, $d$-ideals in $G(X)$, noting that the domain of definition of $v$ may be 
transferred from $M(X)-\{0\}$ to $G(X)$. By Theorem 2 these $d$-ideals are $t$-ideals and we thus obtain an order preserving homomorphism $v^{*}$ of $G(X)$ onto $\Gamma$ such that inverse images (relative to $v^{*}$ ) of $t$-ideals in $\Gamma$ are $t$-ideals in $G(X)$. This latter property may also be expressed by the inclusion $v^{*}\left(A_{t}\right) \subset\left(v^{*}(A)\right)_{t}$. Applying this inclusion to the set $A=\{a, b\}$ we get $v^{*}\left((a, b)_{t}\right) \subset\left(v^{*}(a), v^{*}(b)\right)_{t}$. Since $G(X)$ and $\Gamma$ are GCD-groups, we have $(a, b)_{t}=(a \wedge b)$ and $\left(v^{*}(a), v^{*}(b)\right)_{t}=\left(v^{*}(a) \wedge\left(v^{*}(b)\right)\right.$, and the above set inclusion reduces to $v^{*}(a \wedge b) \geqq v^{*}(a) \wedge v^{*}(b)$. Since $v^{*}$ is order preserving, it follows that $v^{*}(a \wedge b) \leqq v^{*}(a) \wedge v^{*}(b)$ and hence also $v^{*}(a \wedge b)=v^{*}(a) \wedge v^{*}(b)$, showing that $v^{*}$ is a homomorphism of GCD-groups. Conversely, we can associate to any homomorphism of GCD-groups $v^{*}: G(X) \rightarrow \Gamma$ a valuation of $M(X)$ which is positive on $A(X)-\{0\}$ and which has $\Gamma$ as its value group. This establishes the one-to-one correspondence alluded to in the theorem (which may also be formulated for the equivalence classes of valuations instead of the valuations themselves).

Finally, we ought to mention that it could perhaps be interesting to look into the case of several complex variables from the same view-point as we have advocated above. The natural generalization of an open Riemann surface is here a Stein manifold. The divisibility theory of the ring of entire functions on a Stein manifold cannot be described as forcefully and precisely as in the case of the Weierstrass-Florack theorem. In spite of the validity of a special form of the Wedderburn-Helmer theorem (see [9], p. 244), the general existence of greatest common divisors cannot be proved, i.e., the group of divisibility of a Stein manifold is generally not a GCDgroup. However, this group possesses a sufficient degree of integral closure ( $s$-closure) in order to be embedded as an ordered subgroup of a GCD-group. Hence it can also be isomorphically injected into a direct product of totally ordered groups. One of Hej Iss'sa's main results in [12] is a further substantial precision of this fact. He shows that the ring of all holomorphic functions on a (normal) Stein variety $X$ is equal to the intersection of all the discrete rank 1 valuation rings of the field of all meromorphic functions on $X$.

\section{References}

[1] Alling, N. L.: Global ideal theory of meromorphic function fields. - Trans. Amer. Math. Soc. 256, 1979, 241-266.

[2] Arnold, J.: Ideale in kommutativen Halbgruppen. - Rec. Math. Soc. Moscou, 36, 1929, $401-407$.

[3] Aubert, K. E.: Localisation dans les systèmes c’idéaux. - C. R. Acad. Sci. Paris Sér. A-B 272, 1971, A465-A468.

[4] Aubert, K. E.: Divisors of finite character. - Ann. Mat. Pura Appl. 133, 1983, 327-361.

[5] Banaschewski, B.: Zur Idealtheorie der ganzen Funktionen. - Math. Nachr. 19, 1958, $136-160$.

[6] Bourbaki, N.: Commutative algebra, Chapter 7: Divisors. - Hermann, Paris, AddisonWesley, Reading-Palo Alto-London--Don Mills, 1972.

[7] Clifford, A. H.: Arithmetic and ideal theory of commutative semigroups. - Ann. of Math. $39,1938,594-610$. 
[8] FloRACK, H.: Reguläre und meromorphe Funktionen auf nicht geschlossenen Riemannschen Flächen. - Schriftenreihe Math. Inst. Univ. Münster 1, 1948, 1-34.

[9] Gunning, R. C., and. H. Rossi: Analytic functions of several complex variables. - PrenticeHall, Inc., Englewood Cliffs, N. J., 1965.

[10] Helmer, O.: Divisibility properties of integral functions. - Duke Math. J. 6, 1940, 345-356.

[11] Henriksen, M.: On the prime ideals of the ring of entire functions. - Pacific J. Math. 3, 1953, 711-720.

[12] Iss'sA, H.: On the meromorphic function field of a Stein variety. - Ann. of Math. 83, 1966, 34-46.

[13] Jensen, C. U.: On characterizations of Prüfer rings. - Math. Scand. 13, 1963, 90-98.

[14] LoRenzen, P.: Abstrakte Begründung der multiplikativen Idealtheorie. - Math. Z. 45, 1939, $533-553$.

[15] Wedderburn, J. H. M.: On matrices whose coefficients are functions of a single variable. Trans. Amer. Math. Soc. 16, 1915, 328-332.

University of Oslo

Institute of Mathematics

Blindern

Oslo 3

Norway

Received 4 April 1984 